This item was submitted to Loughborough's Research Repository by the author.

Items in Figshare are protected by copyright, with all rights reserved, unless otherwise indicated.

\title{
Review of lightweight deflectometer for routine in situ assessment of pavement material stiffness
}

PLEASE CITE THE PUBLISHED VERSION

PUBLISHER

(C) National Academy of Sciences

LICENCE

CC BY-NC-ND 4.0

REPOSITORY RECORD

Fleming, Paul R., Matthew W. Frost, and John Peter Lambert. 2019. "Review of Lightweight Deflectometer for Routine in Situ Assessment of Pavement Material Stiffness". figshare. https://hdl.handle.net/2134/3523. 
This item was submitted to Loughborough's Institutional Repository by the author and is made available under the following Creative Commons Licence conditions.

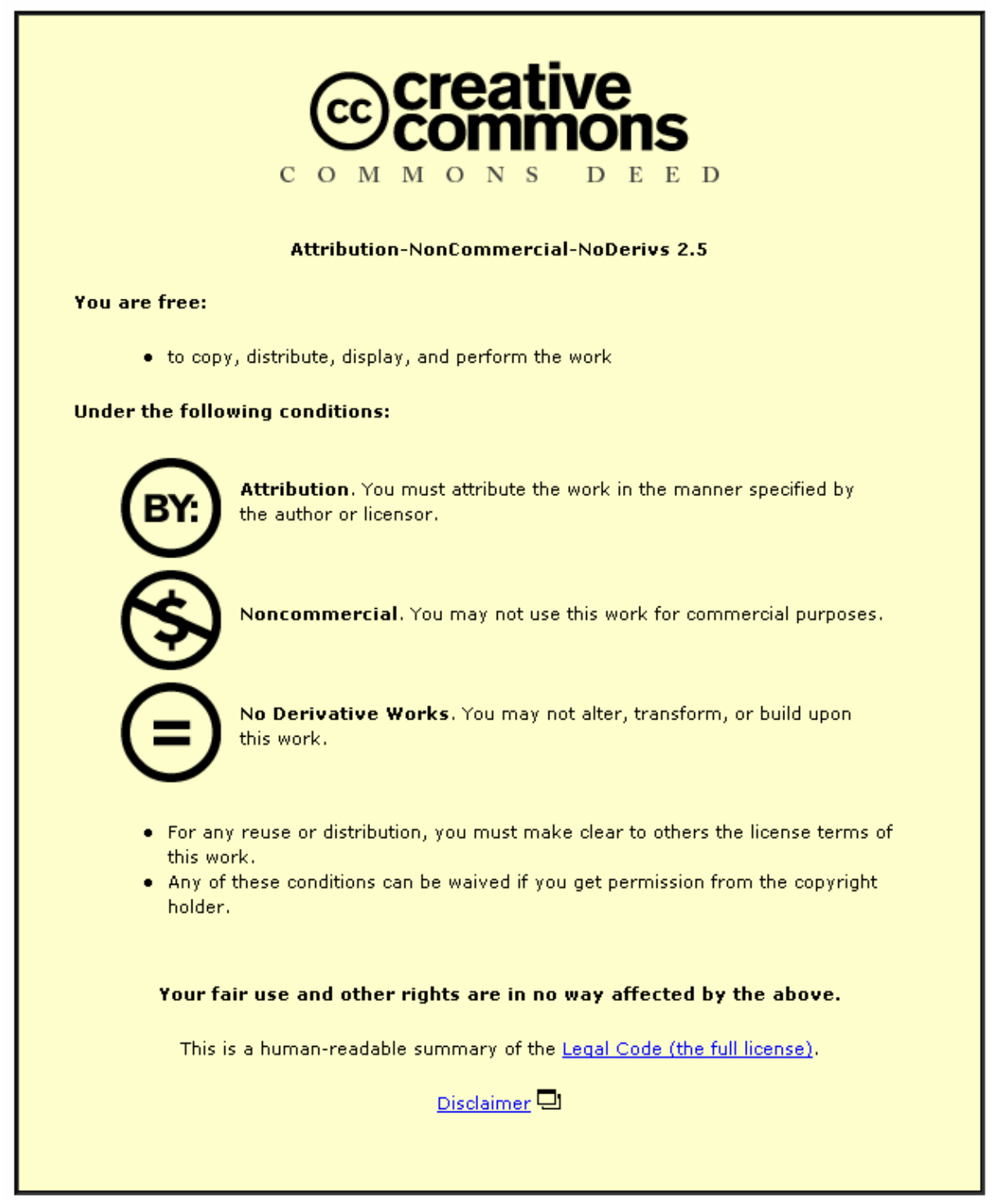

For the full text of this licence, please go to: http://creativecommons.org/licenses/by-nc-nd/2.5/ 


\section{A REVIEW OF THE LIGHTWEIGHT DEFLECTOMETER (LWD) FOR ROUTINE INSITU ASSESSMENT OF PAVEMENT MATERIAL STIFFNESS}

Paul, R, Fleming,

Senior Lecturer

Department of Civil and Building Engineering,

Loughborough University,

Loughborough,

Leicestershire,

LE11 3TU, UK,

+44 (0)1509 222616, Fax +44 (0)1509 223981,

p.r.fleming@lboro.ac.uk (corresponding author)

Matthew, W, Frost,

Lecturer

Department of Civil and Building Engineering,

Loughborough University,

Loughborough,

Leicestershire,

LE11 3TU, UK,

+44 (0)1509 228306, Fax +44 (0)1509 223981,

m.w.frost@lboro.ac.uk

John, P, Lambert,

Researcher

Department of Civil and Building Engineering,

Loughborough University,

Loughborough,

Leicestershire,

LE11 3TU, UK,

+44 (0)1509 222637, Fax +44 (0)1509 223981,

j.p.lambert@lboro.ac.uk

Word count $(5$ figures, 1 table $)=7359$, Paper No.07-1586 Submitted 14/11/06 


\begin{abstract}
The use of a portable lightweight deflectometer (LWD) for construction quality control or material investigation for earthworks and road construction is increasing around the world. This paper reviews the LWD as a field evaluation tool, discusses the test variables and data quality and concludes both on its usefulness and also its limitations for a variety of earthwork and road assessment scenarios. The paper aims to provide a state of the art reference document for LWD users, consultants, material specifiers, contractors and clients. It reviews data from road foundations (subgrades, granular capping and sub-base) and fully constructed in-service (thinly surfaced) roads, to demonstrate the flexibility of the LWD but also show that its determination of 'stiffness modulus' may differ from that of the conventional Falling Weight Deflectometer (FWD) to a varying extent.

A series of laboratory investigations are presented that demonstrate the sensitivity to uniformity of plate/surface contact, and also limitations in the interpretation of peak displacement from the device. In conclusion, a good understanding of the device workings and careful specification of the test variables are required to both analyse the data correctly and permit comparison between data sets. The device is concluded to be a useful and versatile field quality control and pavement investigation tool, if an understanding of the device issues is considered by data users.
\end{abstract}

(218 words) 


\section{INTRODUCTION}

This paper reviews the use of portable falling weight deflectometers (PFWDS) and the behaviour of the Lightweight Deflectometer (LWD) in detail. In this paper the term PFWD means any portable device for measuring a stiffness of material insitu, and the term LWD refers to the specific type of PFWD commonly known in Europe as the Prima, manufactured by Carl Bro (previously manufactured by Keros).

The LWD is increasingly used for investigations of road construction materials and quality control. A background to the LWD development, a review of field data with a focus on correlation with the Falling Weight Deflectometer (FWD), and then specific laboratory investigations are presented. This paper is intended for reference by LWD users, consultants, material specifiers, contractors and clients.

\section{BACKGROUND TO THE USE OF LIGHTWEIGHT DEFLECTOMETERS}

Over the past 15 years the UK Highways Agency has sponsored extensive investigations into the development and use of a range of Portable Falling Weight Deflectometers (PFWDs). One of the key aims has been to establish a specification for such PFWD devices to be used as a field compliance tool within a performance based specification for pavement foundation construction, aimed to optimise the use of materials (1). However, previously the introduction of field testing had been restricted by the need for reliable insitu testing methods to measure suitable parameters whilst being robust enough to withstand the relatively harsh site environment. The Lightweight Deflectometer (LWD), has been used extensively by the authors in research into the evaluation of materials in the field, in the laboratory, on fully constructed in-service (thinly surfaced) roads and more recently on artificial sport surface constructions

The FWD has been in use now for over 20 years, including a certain amount of usage on unbound pavement foundations. It is a tried and trusted tool and is seen by many as a standard against which other dynamic plate tests should be judged. By this measure, many authors have reported correlations between the FWD and PFWDs, (discussed later). In essence the LWD currently uses the same or very similar technology to FWD test equipment, with the primary compromise being the reduced load pulse duration and reduced maximum applied force (i.e. lower weights manually lifted) to maintain portability.

The FWD may not be suited to the routine assessment of roads during construction, specifically where access may be restricted for the towing vehicle and trailer, or where the scale and frequency of testing may make it uneconomic. In addition, the cost and oversophistication of the FWD render it less suited to testing the pavement subgrade and foundation layers, as these are typically subject to in-services stresses at the lower end of the FWD stress range, and assessment of the full deflection bowl is not required or reliable. In recent years there has been research into using the PFWDs on full pavement structures for routine assessment and this appears driven by cost. The number of PFWDs and experience with them in the US is considered limited, but is increasing. It is evident that the use of PFWDs is more trusted if they show good correlation to the FWD. Where they have been used as a quality control tool and this has been demonstrated through current FHRP projects such as 'Intelligent Compaction'(2).

However, many questions have arisen about PFWDs, specifically if they are to be used as part of contract control. The focus of much of the work in the UK has been on demonstrating the usefulness and reliability of the LWD through field trials. Little work has been carried out on detailed assessment of the influence of the test device variables on the potential outcomes of the measurements. Such variability assessments have mainly been 
performed on natural materials and these are intrinsically difficult to use to study repeatability and accuracy.

\section{PFWD/ LWD REVIEW}

The LWD has been available for several years, earlier somewhat similar PFWDs for measuring stiffness insitu included the TRL Foundation Tester (TFT) (3), the Natural Vibrations Method (NVM) (4), Leichtes Fallgerät from Zorn (known as the 'German Dynamic Plate' or GDP) (5), the Soil Stiffness Gauge (also known as the SSG) (5), the Loadman from Finland (4), the ODIN apparatus (4) the Clegg Hammer (4). All these devices have been described in more detail elsewhere, and can be categorised by the load pulse rate and intensity of maximum load (or contact pressure) (4). To summarise, the ODIN (a research prototype) and Clegg (often used for compaction control) comprise rapid undamped impact tests, the SSG (not specified in the UK) and NVM (a research prototype) comprise small devices that measure the response to low energy impulses applied over a range of frequencies. The Loadman and GDP comprise damped impacts of a falling mass onto a bearing plate, (the novelty of the Loadman stemming from its enclosed tube apparatus), however both interpret the impact using an accelerometer rather than directly from a load cell, and in general the interpreted deflection is observed to be less reliable (5). The TFT was a research prototype developed in 1992, and similar in all ways except it had a higher mass bearing plate than the LWD. It was used in research on live sites until 2000, whereupon the LWD became the adopted portable plate test in the UK although it was not formally specified in detail in UK guidance until recently (6). Whilst all the portable devices have their advantages and disadvantages the LWD has emerged as the most acceptable tool retaining some flexibility in test protocol, such as variable plate size, load magnitude, and data collection, with the added and important factor that it most closely resembles the loading rate and area of a (single) moving wheel (4), and functions similarly to the FWD.

The LWD is shown schematically in Figure 1. The test variables include drop weight, drop height, load contact area, rate of loading and the number of geophones (up to two extra geophones can be connected to allow a limited deflection bowl to be measured). The stiffness modulus (E) from the LWD is calculated using equation 1.

One particularly important aspect when considering the measurements made is the interpretation of the deflection under load. In general the device software integrates the geophone (velocity transducer) signal to determine the maximum (or peak) deflection value. This has two important ramifications, the first being that under test the peak deflection may not occur at the same instant as the peak load (Figure 2), and usually does not, specifically for lower stiffness materials. The second is that the maximum deflection may include an element of permanent/plastic deflection in addition to recoverable/elastic deflection. This depends upon the 'strength' of the materials under test, and the efficacy of the contact between the geophone foot and the material under test. Thus it is apparent that the term 'elastic' stiffness (E) should be applied very carefully to all PFWDs, and the use of such 'elastic' values in elastic pavement analysis needs careful consideration.

$$
\mathrm{E}=\frac{\mathrm{A} \cdot \mathrm{P} \cdot \mathrm{r} \cdot\left(1-v^{2}\right)}{\mathrm{d}} \quad(\mathrm{MPa})
$$

Where:

$\mathrm{E}=$ stiffness modulus $(\mathrm{MPa})$

$\mathrm{A}=$ plate rigidity factor, default $=2$ for a flexible plate, $\pi / 2$ for a rigid plate.

$\mathrm{P}=$ maximum contact pressure $(\mathrm{kPa})$ 
$\mathrm{r}=$ plate radius $(\mathrm{m})$

$\mathrm{v}=$ Poisson's ratio (usually in the range 0.3-0.45 depending on test material type)

$\mathrm{d}=$ peak deflection $(\mathrm{mm})$

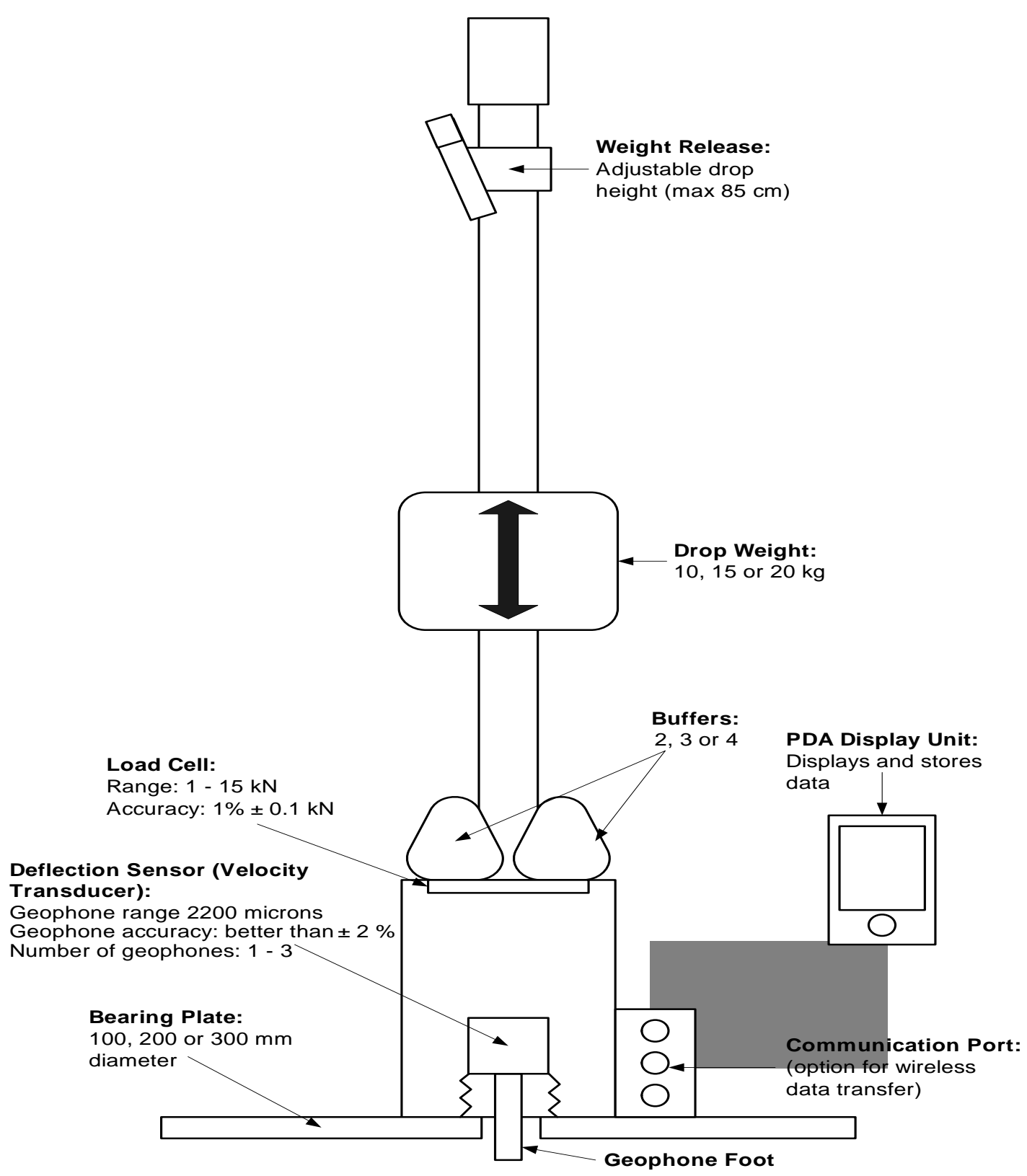

\section{FIGURE 1. A Schematic of a Lightweight Deflectometer (LWD) Showing the Test} Variables

The impetus for developing smaller portable FWDs has largely been driven by the slow and cumbersome nature of conventional Static Plate Bearing Tests (SPBT) for insitu stiffness. However, there is much to be said for the SPBT method (7) which allows a range of stresses to be applied and the deflection measured both during the loading and the unloading phase, thus allowing a better determination of elastic/recoverable deflections. Cycles of load-unload can be applied to aid determination of the adequacy of compaction. 


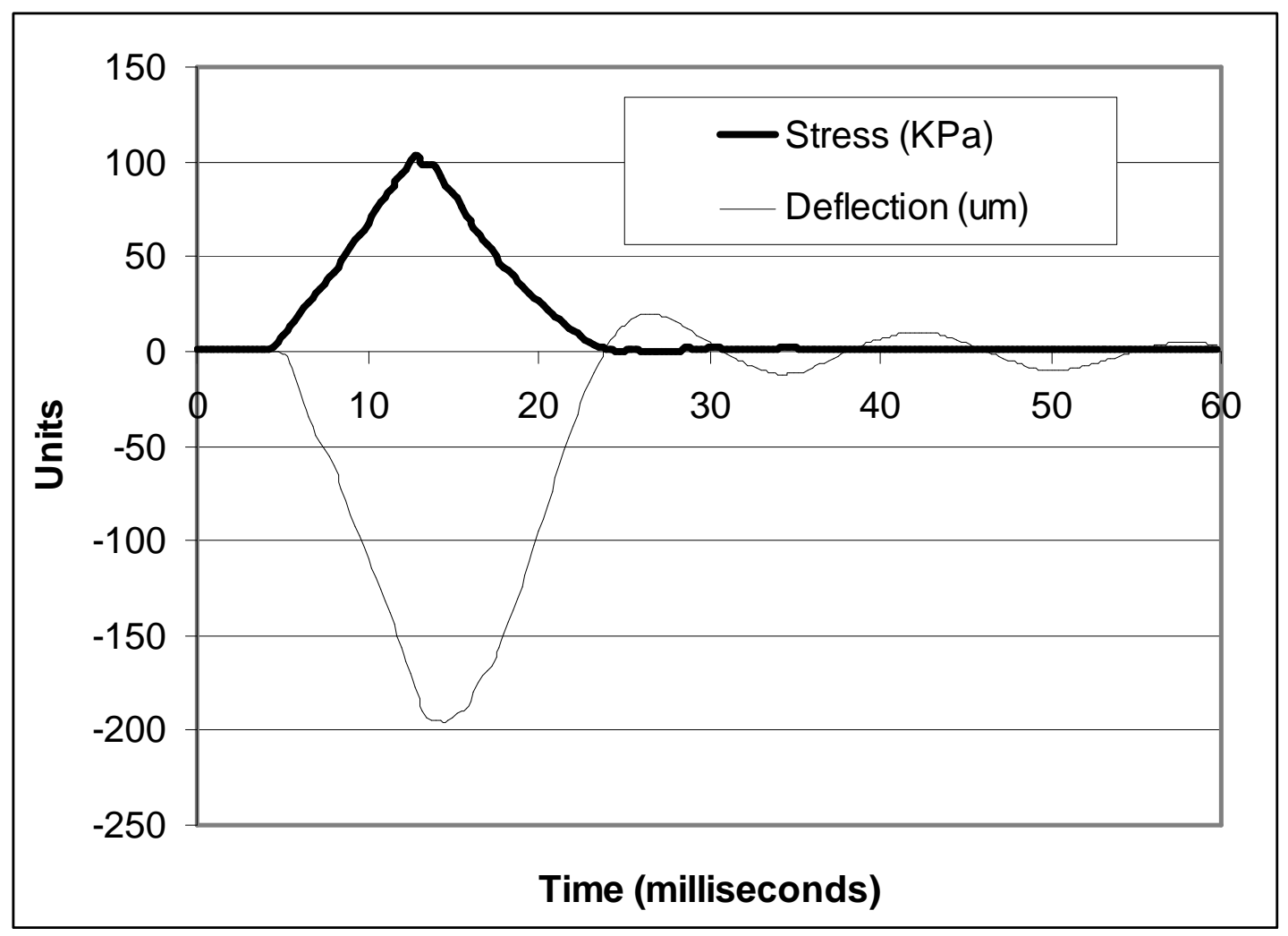

FIGURE 2. Example of LWD Output from a Laboratory Test on $400 \mathrm{~mm}$ of a Well Graded Crushed Rock (Granodiorite). The Drop Height was Controlled to Achieve a Contact Stress of $100 \mathrm{kPa}(300 \mathrm{~mm}$ Diameter Plate Size).

\section{LWD FIELD DATA}

\section{Road Foundations}

There have been several publications showing correlations between the range of Portable FWDs and the full scale FWD. This section briefly reviews the findings from several publications, to illustrate the range of correlation coefficients determined, and also to identify any significant device related comments, such as the zone of applied stress from the device or material stress dependency observed.

Fleming et al (8) looked in more detail at the GDP and TFT. The TFT gave a correlation range of 0.8 to 1.4 FWD (200 tests), from tests on a range of pavement foundations constructed of well-graded granular materials at live sites. The TFT and LWD were compared extensively at a bespoke field trial (based on a $500 \mathrm{~mm}$ thick granular layer over silty clay) and gave a correlation TFT $=0.96$ LWD and $\mathrm{R}^{2}$ of 0.92 (25 tests). The LWD was compared to the FWD at a site of $450 \mathrm{~mm}$ granular capping over silty clay and found $\mathrm{LWD}=0.97 \mathrm{FWD}$, and $\mathrm{R}^{2}$ of 0.6 (25 tests). It was concluded that such device correlations were likely therefore to be site, material and device specific, and this has been confirmed in more recent work to develop the new UK Performance Based Specification, and highway foundation design guidance (6). For example, on a $260 \mathrm{~mm}$ thickness of lime and cement treated clay subgrade, LWD $=1.21 \mathrm{FWD}, \mathrm{R}^{2} 0.77$ (25 tests). At another site with a standard granular foundation $(225 \mathrm{~mm}$ well graded crushed rock over a granular subgrade) for several sections the range was $\mathrm{LWD}=0.8$ to $1.3 \mathrm{FWD}$, with an $\mathrm{R}^{2}$ of 0.5 or lower (169 tests). However, this latter site was quite wet and the stiffness values were generally low relative to other sites evaluated. 
Other research reported on comparisons between PFWDs (5), showed a strong stress dependency (of the reported stiffness for the LWD (and TFT) on a typical granular foundation materials, with changing device applied stress (i.e. by changing drop height). However little stress dependency was observed with the FWD, albeit testing at a higher stress range. The very lowest stress achievable with the lighter version of the FWD is around $100 \mathrm{kPa}$, which is the normal applied stress for typical LWDs.

Nazzal et al (9) carried out comparative testing of the FWD, LWD and SSG on live construction sites and in a accelerated load test facility. They showed a general relationship of FWD=0.97PFWD. In addition, from the controlled laboratory data it was concluded that LWD stressed to a depth of $270-280 \mathrm{~mm}$, or approximately one load plate diameter (this depth of stressing was significantly deeper than the depth assessed as stressed by the SSG).

Peterson et al (2) reviewed the use of PFWD devices to provide site quality control for compaction. They found that the LWD stressed to between 0.1 and $0.3 \mathrm{~m}$ deep, and this approximated to $3000 \mathrm{~cm}^{3}$ of soil volume being stressed the was similar to the volume assessed as stressed by Static Plate Tests and the SSG. They also concluded that the modulus appeared to be dependent on the stress applied (i.e. drop height), and that this was more of a factor for the LWD than for the FWD.

In addition to the correlation between devices, it is of interest to observe the variability of stiffness with position along a section of notionally the same construction (i.e. materials, layer thickness and water content). In the UK data has been analysed in detail and a general pattern has emerged, which is useful for selecting the appropriate frequency of testing and also the setting of target values for quality assurance and quality control on site. In general, the variability in any one trial section can be usefully reported as the Coefficient of Variance $(\mathrm{CoV})$, which is the ratio between the standard deviation and the mean for a test section. The reported range of CoV observed was 25-60\% for FWD and LWD on predominantly fine grained subgrades (5), perhaps due to variation in water content. For granular capping layers in the CoV range observed was 10-40 \% (higher when wet). For subbase materials (highly specified well graded crushed rock) the CoV observed was typically less than $15 \%$ (higher on very wet sites).

\section{Thinly Surfaced Roads}

The use of PFWDs on fully constructed roads is also attracting some interest. Steinert et al (10) looked at the potential use of LWD for monitoring and intervention/traffic load control associated with spring-thaw seasonal variations. They produced a comparison between the LWD and FWD on a variety of sites at differing times of the year. The relationship between the LWD and FWD was shown to vary with reducing asphalt thickness. In addition the goodness of fit between the data showed a better correlation $\left(\mathrm{R}^{2}=0.87\right)$ on thinner asphalt layers. The correlations were $\mathrm{LWD}=1.33 \mathrm{FWD}$ for the thin, and $\mathrm{LWD}=0.75 \mathrm{FWD}$ for thicker asphalt (whereby the $\mathrm{R}^{2}$ reduced to 0.56 ). However, the authors reported that excluding very high measured stiffness results ( $>4000 \mathrm{MPa}$, which equates to LWD deflection of less than 7 microns) generally improved the $\mathrm{R}^{2}$ to as high as 0.8 . However, it is not clear from the work, whether the comparative measurements were at the same contact stress, or to what extent this may influence the results. The LWD data reported gave stiffness modulus on the asphalt, for thickness $127-180 \mathrm{~mm}$ of $645-503 \mathrm{MPa}$ for the period after thawing. They recommended a large drop mass and high drop height for testing asphalts, to ensure there was some influence from the sub-base in the measured deflection.

In a study by the authors, the LWD was compared to the FWD (both using a $300 \mathrm{~mm}$ diameter load bearing plate) on a thinly surfaced construction (similar to a car park design in the UK, but in this case applied to the foundation for a synthetic hockey pitch) (11). LWD testing was carried out on the sub-base and asphalt, and the FWD was utilised on the asphalt 
only. A total of fifty test positions were evaluated on a regular grid at $10 \mathrm{~m}$ intervals, over a rectangular area of approximately $5000 \mathrm{~m}^{2}$. The sub-base was specified to be $250 \mathrm{~mm}$ thick and porous to allow drainage. The asphalt was $65 \mathrm{~mm}$ of porous asphalt placed in two lifts. The evaluation showed the sub-base to be poorly compacted with an average stiffness modulus of $30 \mathrm{MPa}$, with a range of $5-81 \mathrm{MPa}$, and a CoV of $31 \%$ (50 tests). Similar low stiffness results were achieved with the other PFWDs used at this site (the GDP and TFT). The FWD weights and drop height were reduced to achieve $100 \mathrm{kPa}$ peak contact stress to match the LWD, with the central geophone used to determine the overall pavement modulus . The FWD gave a stiffness modulus average of $61 \mathrm{MPa}$, and the CoV was $14 \%$ (50 tests), which is assessed as very uniform in the light of the previously presented data. The LWD, on the asphalt, gave an average stiffness of $110 \mathrm{MPa}$, with a $\mathrm{CoV}$ of $17 \%$ (50 tests), and was also deemed uniform. In addition, in this instance the GDP gave a much higher average stiffness modulus of $140 \mathrm{MPa}$ on the asphalt, this relationship had not been observed before, $\mathrm{GDP}=2.26 \mathrm{FWD}$ with an $\mathrm{R}^{2}$ of 0.63 (50 tests).

These data on thin asphalt layers were seen to be particularly interesting as it appeared that the relationship between the LWD and FWD was particularly affected by the relatively thin and stiff upper layer. It is considered that the primary reason for the LWD (and GDP) recording a much lower deflection (and hence higher modulus) is that the depth of significant stressing is smaller than for the larger FWD. This is considered to be due to the rate of loading, whereby the FWD applies the load over a longer period of up to 35 milliseconds, and the portable devices deliver the load pulse over a duration of approximately 15-20 milliseconds (on the asphalt this was in the range 16-17milliseconds). In addition the FWD trailer weight does apply some preload to the material through the wheels, and the bearing plate assembly is also much heavier than for the LWD.

The ratio of stiffness between the upper and lower layers here may have exacerbated these errors, as the sub-base was clearly of low stiffness, and the stiffness ratio (asphalt to sub-base) could have been as high as 10 (assuming 3GPa for the asphalt layer). In road foundations during construction the typical stiffness ratio of layers is usually assumed at up to 3 (6). In many cases, the ratio of subgrade stiffness to granular capping and sub-base stiffness is less if construction occurs in a period of good weather, as the (fine grained) subgrade is often able to sustain some suctions and as a consequence appear much stiffer when tested.

The brief field data, presented here, again show that there is not a unique relationship between the LWD and the FWD, which is perhaps not surprising when one considers the different loading rate. The material layer thickness and stiffness ratio may be the primary factor in determining the magnitude of the correlation between any devices. However, the important issues of test device repeatability and defining acceptable variability on a site need to be considered.

For a series of tests on a uniform construction, expressing the data in the form of $\mathrm{CoV}$ is considered useful, and the smaller the $\mathrm{CoV}$ the better. It is also clear that the more uniform the placed material and its compacted state the lower the CoV. However, the question remains as to whether the variability in measured stiffness is wholly due to the materials under test and their state (such as water content, density, and thickness), or whether there is variability due to the device. One important, and obvious, aspect is the integrity of the seating of the bearing plate on the ground, especially for the lighter LWD (relative to the FWD). It is difficult on site to ensure very uniform contact of the plate, especially on coarser aggregates as further discussed below.

\section{LABORATORY EVALUATIONS}

Controlled laboratory research has considered the potential influence of the many test variables described above. To assess the repeatability and reliability of the devices. Over a 
series of smaller projects, the following variables have been investigated, including buffer temperature, plate diameter, drop height, drop weight, geophone/loading plate attachment (fixed/loose), use of extra geophones (backanalysis), and plate-surface contact. The buffer temperature effects and plate-surface contact effects are described in detail below.

In addition, prior to use the devices need to be checked carefully, and a method has been developed to carry out tests on a stiff repeatable substrate, such as the laboratory concrete floor to check the deflection and load pulse before any testing. Geophones have also been swapped between similar devices and compared such that any damage to a transducer can be quickly identified.

\section{Buffer Temperature}

The influence of temperature on the LWD buffers and hence loading characteristics was evaluated in the laboratory. The rubber buffers were evaluated at the three different temperatures, and measurements were made with the LWD on the rigid laboratory floor (calibration spot) with the mass dropped from the same height each time. Two buffers were used, and the data recorded for 10 repeat impacts. The stiffness of the floor is relatively high compared to the majority of field test sites, the floor giving a stiffness of 2440MPa. However, regardless of buffer temperature the stiffness remained effectively constant, the only readily observable change was in the reported length of the load pulse, which was seen to increase with buffer temperature from 18 to 20 milliseconds (Table 1). This would be expected as the buffers soften slightly when heated.

TABLE 1. Effect of LWD Buffer Temperature on Output Data From Tests on a Laboratory Concrete Floor

\begin{tabular}{ccccccc}
\hline Temperature & & Force(kN) & Press(kPa) & Pulse Time(ms) & D1(um) & E1(MPa) \\
\hline Ambient & Mean & 6.91 & 97.80 & 19.03 & 9.02 & 2442.21 \\
$21 \pm 2{ }^{\circ} \mathrm{C}$ & SD & 0.20 & 2.79 & 0.08 & 0.34 & 68.27 \\
& COV & 2.86 & 2.86 & 0.42 & 3.80 & 2.80 \\
\hline Heated & Mean & 6.84 & 96.75 & 20.20 & 9.31 & 2338.49 \\
$80 \pm 10^{\circ} \mathrm{C}$ & SD & 0.02 & 0.24 & 0.11 & 0.08 & 23.94 \\
& COV & 0.25 & 0.25 & 0.52 & 0.85 & 1.02 \\
\hline Cooled & Mean & 6.70 & 94.80 & 18.15 & 8.70 & 2453.41 \\
$-10 \pm 10^{\circ} \mathrm{C}$ & SD & 0.07 & 0.94 & 0.41 & 0.12 & 34.27 \\
& COV & 0.99 & 0.99 & 2.27 & 1.37 & 1.40 \\
\hline
\end{tabular}

\section{Plate-Surface Contact}

Recent work (12) has evaluated the LWD for use in a material approval laboratory test for coarse granular materials using a large test mould. The work is not presented herein, however, as part of this work an investigation was made into the influence of the platesurface contact, and the effectiveness of the LWD software interpretation of peak displacement. This was aimed to evaluate if a 'bad' drop could be recognised in the device force and deflection versus time signal (which is displayed on the device readout and can be saved for subsequent analysis in a spreadsheet).

The upper surface of a well compacted $400 \mathrm{~mm}$ layer of a coarse well graded crushed rock was disturbed by removing the material in one area to approximately a depth of 5$10 \mathrm{~mm}$. The LWD was then positioned in several locations deliberately causing poor uniformity of plate contact. Subsequently, the uneven area was carefully filled with a single size sand (nominal size $1.15 \mathrm{~mm}$, as is recommended for improving contact for static plate testing (7)). Two typical pressure-deflection traces from the last (of six) LWD impacts are 
shown in Figure 3 and clearly demonstrate the improvement in the regularity of the shape of the trace with improved contact. If the raw data are reviewed on the hand-held unit in real time, (whereby load and deflection are shown against time), the 'poor' contact is observed to be a small early peak in the deflection trace and is further discussed below. In addition, during the test there was observed to be some bounce and/or horizontal movement of the apparatus, and some vibration back through the device again suggesting poor uniformity of contact. However, the judgement of poor contact is subjective, and the on-site assessment of impact quality is difficult. In this case, for example, the reported stiffness was $75 \mathrm{MPa}$ for the 'poor' contact and $145 \mathrm{MPa}$ for the 'good' contact. On site such variability is not unknown, and many possibilities exist within an end-product specification whereby the acceptance or rejection of individual test data based on the user perception of impact quality could be contractually difficult. This example is clearly a rather extreme case, but shows that there is merit in examining in more detail the data signal generated at the time of test, and possibly the development of a routine within the software to identify poor quality impact data. It is interesting to note that during the development and evaluation of the TFT (13) the site measurements were reportedly of poorer repeatability where the aggregate had become segregated at the surface, in particular where there was a lack of fines.

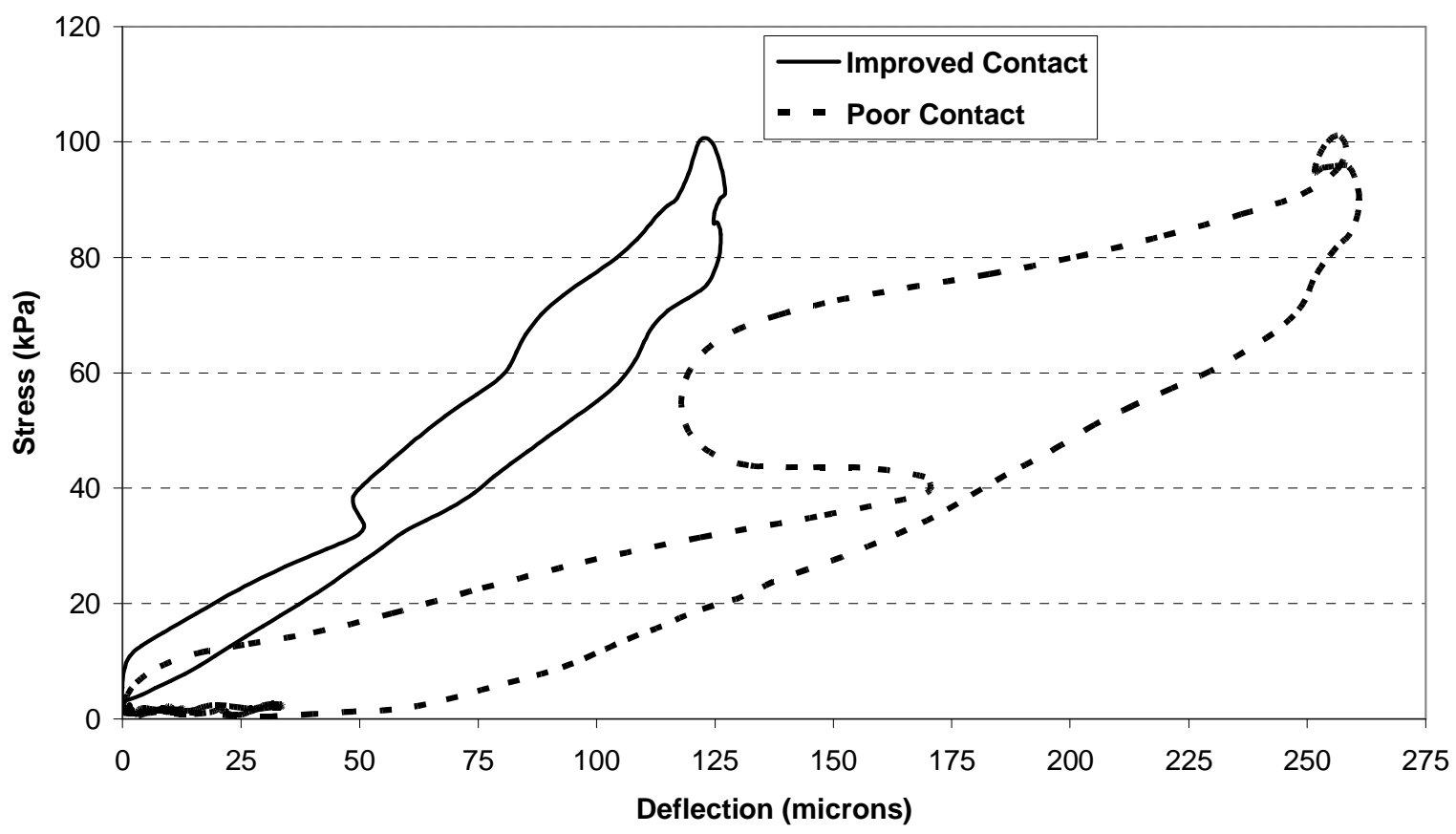

FIGURE 3. The Effect of Poor and Improved Plate-surface Contact on the LoadDeflection Trace for a 300mm Diameter Plate Impact Test on a Well Graded Crushed Rock Aggregate.

Another consideration is the interpretation of the maximum deflection, and the use of the deflection time trace to indicate poor quality impact or poor quality material. The same $400 \mathrm{~mm}$ depth of granular material was excavated to a new level, removing around $100 \mathrm{~mm}$ of aggregate. A well graded (slightly gravely) sand, known as concrete river sand, was then applied in a $100 \mathrm{~mm}$ layer and compacted in stages, with LWD tests carried out at each stage. Figure 4 shows the effect of the increasing compaction on the sand layer, and the 
consequence on the deflection displayed by the LWD. Of particular interest is that the peak deflection recorded (and used in the stiffness calculation) and displayed on the hand held unit is the maximum deflection, regardless of its position (in time) relative to the peak load/contact stress (the stress pulse trace is shown with a peak at around 9 milliseconds). Thus the 'peak' deflection for each of the tests carried out on the 'loose', '2 passes' and ' 4 passes' is erroneously reported as the peak to the right of the (smaller) peak deflection that occurs at a similar instant to the maximum impact stress. In these cases the stiffness reported is a falsely low value. These traces additionally show that the deflection, post impact indicate some substantial permanent deformation was occurring, and this was observed after the removal of the device (see Figure 5). This is considered particularly significant as it confirms that the peak deflection can potentially contain both significant plastic and elastic components. The peak deflection represents the total deflection under loading and not that which is recoverable, and it is this total that is included in the stiffness calculation in equation 1. After 6 passes the deflection trace is more uniform however the effect of providing an improved more level surface is clearly shown by the ' 6 passes plus improved contact' trace. It is also important to note that the peak displacements that occur soon after the peak contact stress are very similar in magnitude for the two well compacted states. Thus the benefits of observing the raw data is clearly evident in the case of this well graded sand.

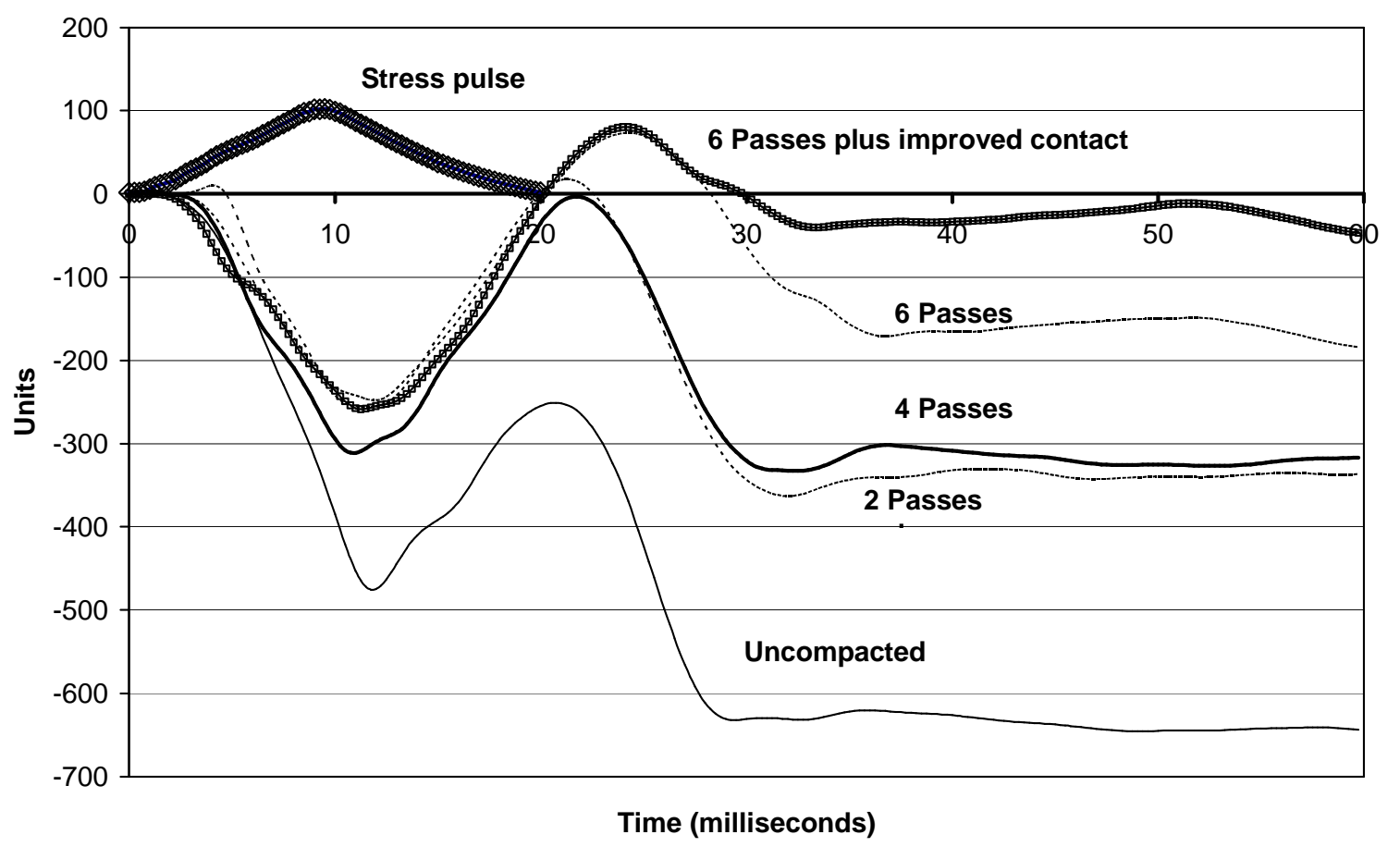

\section{FIGURE 4. LWD Deflection Traces for a Sand Layer with Increasing Compactive Effort.}

\section{Geophone-surface Contact}

Figure 5 shows the sand surface after removal of the LWD. Two important observations can be made, firstly that there is significant permanent deformation under the $300 \mathrm{~mm}$ bearing plate (this from six consecutive impacts). The second is that the geophone contact area (in the centre) is very disturbed. The geophone foot is narrow, and has a relatively stiff spring inside the housing to maintain contact between the geophone and test surface which has caused the disturbance. This disturbance is not uncommon, in the authors' experience, on softer subgrades and some granular materials. It is not clear, however, how much of this observed 
permanent deformation is recorded during an impact. It may be sensible to use a larger diameter geophone 'foot' for weaker materials. This was considered during the development of the TFT, whereby a $25 \mathrm{~mm}$ diameter foot was found to be appropriate (early versions of the LWD also had a larger foot). Edge contact effects on large particles were also thought to occur with a larger foot also, however, and this is an area worthy of further work.

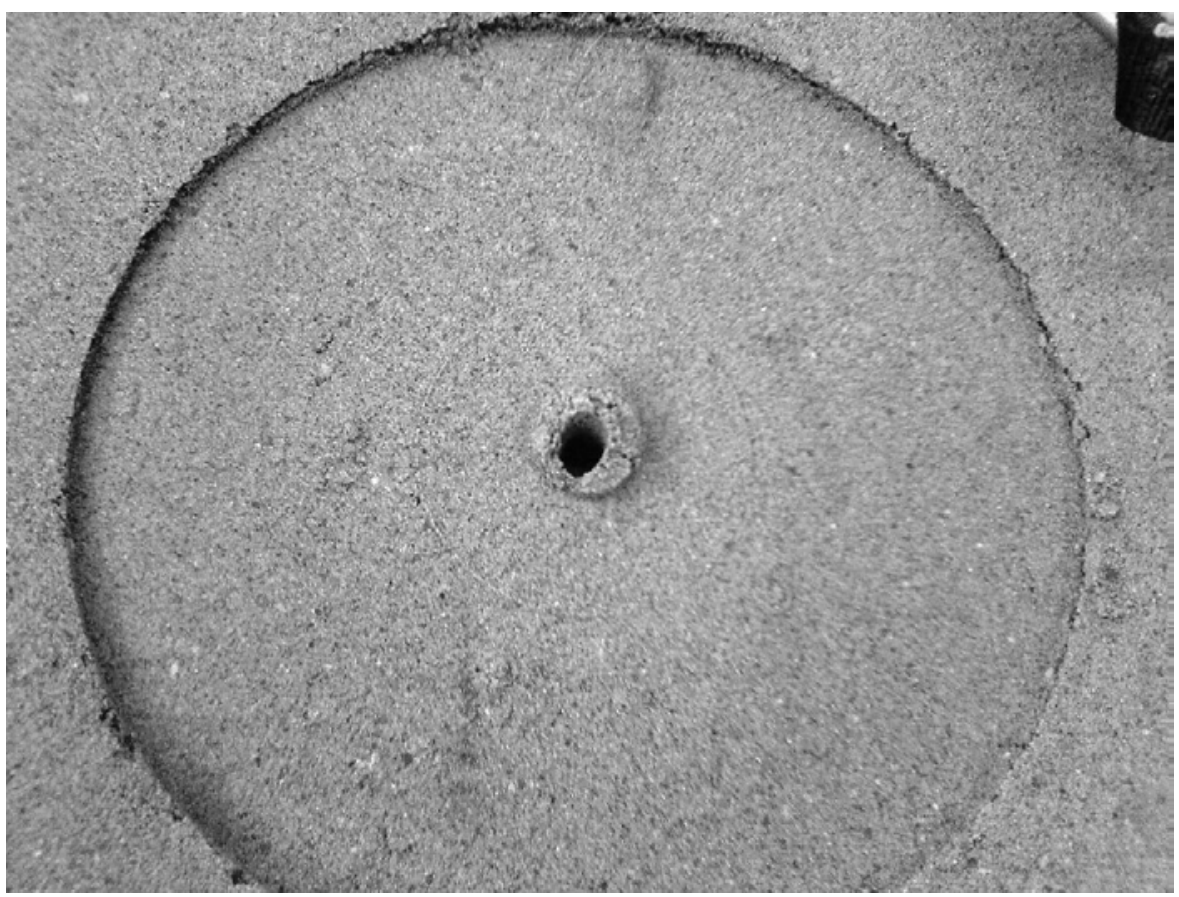

FIGURE 5. The Effect of Repeated LWD Impacts on the Sand Layer, Showing Permanent Deformation Under the Plate and Disturbance Under the Geophone Foot.

\section{DISCUSSION}

The field and the laboratory data available raise several points regarding the data integrity of the LWD. It is clear that the LWD is a versatile tool used to aid engineering decisions related to structural integrity of a wide range of road construction materials. However, it is also clear that to provide high quality data a good understanding of its workings and potential limitations is required.

The site data clearly shows that, in general, a correlation of the LWD to the (accepted) FWD is achievable. The differences in self weight, and in particular rate of loading, may account for the site specific correlations that arise. However, there is always likely to be an issue of variability of site data, and to use the device as a quality control tool measuring absolutes this variability needs to be reduced or allowed for in any analysis perhaps through initial correlation with the FWD at that site or an acceptable CoV range.

One way to improve the data quality may be to ensure the test is carried out as carefully as possible at each location, especially seating, and to interrogate the signal traces to look at the quality and repeatability of the recorded data. The site procedures may need to include the use of a thin sand layer applied to provide a level surface to distribute the impact pressure more uniformly. The selection of appropriate test positions is of course sensible (but can be contentious on sites). Perhaps three repeat tests at immediately adjacent positions to represent one location, and its average stiffness reported, may be suitable. The integrity of the signal traces is perhaps still at the judgement stage, but the data presented have shown that the deflection-time history may be useful in indicating poor contact, and that any deflection 
that occurs well after the peak load should be ignored in any determination of a stiffness value. Second peaks of deflection could be an indicator of significant compaction occurring during testing. The interpretation of the deflection as indicating 'elastic' behaviour is clearly erroneous on many granular or cohesive materials, and thus the use of the LWD derived stiffness modulus in layered elastic analysis should be tempered by experience and a careful review of appropriate values from fieldwork.

The current advice ( 6 ) in the UK defines the acceptable PFWD equipment for use as a quality control method as, "capable of delivering a load pulse of peak magnitude in the range $4-15 \mathrm{kN}$ of total duration $15-60$ milliseconds to a rigid circular plate of $300 \mathrm{~mm}$ diameter. Both the applied load and transient deflection shall be measured directly on the tested surface. The deflection transducer shall be capable of measuring deflections of up to 2000 microns. A contact stress of $100 \mathrm{kPa}$ is recommended, with three seating drops and then three recording drops which are averaged and the stiffness modulus reported".

In addition, it is proposed that a demonstration/trial area is used to validate the design, materials and methods for any performance approach (6). For Permanent works, insitu stiffness tests are stipulated at $20 \mathrm{~m}$ intervals in each lane/carriageway (staggered by $10 \mathrm{~m}$ ). A minimum insitu stiffness value is set by the designer prior to the works as one target. In addition, a running average stiffness of six test results must be above a second target value, also set by the designer. The targets are set based upon accepted (tabulated) values, or laboratory derived stiffness data, and then proved in trials (problems found in the trial permit redesign and material selection changes). However, the document also states that the LWD may not be suited to thicker foundations, and in these cases the FWD is recommended.

There is, however, no clear description of the on-site test protocol for using the device or for analysis of the data, other than the number of drops (six in total). Thus there is still the possibility that poor quality impact test results may be included in the interpretation and decision making, and no advice is given as to how or when to discard any results.

The best form of testing protocol for any site may be variable, and tailored to suit the materials under test. For example, it would appear that the recommended six drops for each location is adhered to, though whether the first three drops are ignored and the next three averaged, or some other combination (10) is more suited is unknown. The decision as to the drop mass and drop height, and also the number of tests per zone of site area can also be varied and may depend on the complexity of the site and the contractual need to monitor materials and workmanship. In addition, many researchers have reported that the stiffness measured was a function of the applied contact stress and hence the drop height needs to be maintained and/or the stress dependent nature allowed for in the data interpretation, especially where data are compared between different PFWDs. It may also be prudent to consider the use of alternative geophone foot shapes on weaker materials to avoid the disturbance observed.

\section{CONCLUSIONS}

The LWD is a versatile and portable stiffness measuring tool. From the published literature it appears to be increasingly used on a variety of materials/constructions including during construction and in-service (on thinly surfaced roads) around the world.

The correlation of the LWD to FWD stiffness (using the central geophone only) appears to be variable, and perhaps site dependent. The reasons for this are not clear, but may be due to the different rate of loading and the self weight of the bearing plate for the two devices.

The LWD is being used as a FWD substitute, and is specified in recently published UK (draft) road design guidance. 
Several factors affecting LWD data quality have been investigated. Buffer temperature is not considered a significant issue. There is some influence due to nonuniformity of plate contact, and this was observed to be improved by the application of a thin layer of uniform sized sand.

For weaker materials the contact between the geophone foot and material surface was seen to raise concerns over punching failure under the foot, (thought to be a function of the strong spring holding the geophone down in addition to the small foot diameter). This spring stiffness could be reduced, or a larger foot could be used to alleviate this problem.

The geophone peak displacement that is interpreted from the signal, and displayed on the readout, is the actual maximum displacement during the recorded period of measurement. This can lead to errors if the post impact 'peak' value is larger than the peak that occurs around the time of the peak load. The test quality may be evaluated, in part, by assessment of the deflection time trace.

The effect of increasing compaction of a layer of sand showed clearly some interesting behaviour of the deflection-time trace. In its loose and partly compacted state the deflection trace appeared to show significant permanent deflection. When fully compacted or with a carefully prepared contact surface the trace was considered more akin to that expected whereby the deflection returns almost to zero.

\section{ACKNOWLEDGEMENTS}

The authors would like to acknowledge the UK Highways Agency, Scott Wilson Pavement Engineering, the Centre for Innovative Construction Engineering at Loughborough University for past collaborations, and Colin Young for his assistance with some of the field and laboratory work. The opinions expressed are solely those of the authors.

\section{REFERENCES}

1. Fleming P. R., and Frost M. W., Lambert J. P., Sustainable Earthworks Specifications for Transport Infrastructure, presented at the 85th Transportation Research Board meeting, National Research Council, CD-ROM, Washington DC, USA, 2006.

2. Peterson D.L., Siekmeier J., Nelson C.R., and Peterson R.L. Intelligent Soil Compaction Technology, Results and a Roadmap toward Widespread Use, presented at the 85th Transportation Research Board meeting, National Research Council, CD-ROM, Washington DC, USA, 2006.

3. Rogers, C.D.F., Brown, A.J. and Fleming, P.R.; Elastic Stiffness Measurement of Pavement Foundation Layers, Proc. of the 4th Int. Symp. Unbound Aggregates in Roads (UNBAR4), Nottingham University, 1995, pp 271-280.

4. Fleming, P.R., and Rogers, C.D.F. Assessment of Pavement Foundations During Construction, Transport, Proc. Institution of Civil Engineers, 111 (2), 1995, pp 105-115.

5. Fleming P. R., Lambert J. P., Frost M. W., Rogers C. D. F., In-situ Assessment of Stiffness Modulus for Highway Foundations During Construction, 9th International Conference on Asphalt Pavements, Copenhagen, Denmark, August, 2002, pp12, CD-ROM.

6. Highways Agency, Design Guidance for Road Pavement Foundations (Draft HD 25), Interim Advice Note 73, Highways Agency, London, February 2006.

7. BSI. Code of Practice for Site Investigation, British Standard 5950, 1999, BSI, London. 
8. Fleming P R, Frost M W and Rogers C D F, A Comparison of Devices for Measuring Stiffness Insitu", Unbound Aggregates in Road Construction, ed Andrew R Dawson, Balkema, 2000, pp 193-200.

9. Nazzal, M. Abu-Farsakh, M., Alshibli, K. and Mohammed, L., Evaluating the Potential use of a Portable LFWD for Characterising Pavement Layers and Subgrades Geotechncial Engineering for Transportation Projects: Proceedings of Geo-Trans 2004, Los Angeles, US, 2004.

10. Steinert, B.C., Humphrey, D.N., Kestler M.A. Portable Falling Weight Deflectometers for Tracking Seasonal Stiffness Variations in Asphalt Surfaced Roads, presented at the 85th Transportation Research Board meeting, National Research Council, CD-ROM, Washington DC, USA, 2006.

11. Fleming P. R., Dixon N., Lambert J, Young C, Monitoring the Performance of Hockey Pitches During Construction, The Engineering of Sport 4, Kyoto, Japan, 2002, pp. 545-552, ed. S Ujihashi and S Haake, Blackwell.

12. Lambert J. P., Fleming P. R., and Frost M. W., Laboratory Assessment of Coarse Granular Road Foundation Materials presented at the 85th Transportation Research Board meeting, National Research Council, CD-ROM, Washington DC, USA, 2006

13. Nunn, M.E., Brown, A., Weston, D., and Nicholls, J.C.. Design of Long Life Flexible Pavements for Heavy Traffic, TRL report 250, 1997, TRL Limited, London, ISSN 09684107. 